\title{
تصميم وتثغيل دائرة تضمين موقع نبضة لليزر أثباه الموصلات المستخدم في الاتصالات البصرية
}

\author{
سلمى محمد حسين * ؛ رنا اسكامه مهاي * و عمار هادي جريز \\ * قسم العلوم النطبيقية، الجامعة التكنولوجية. \\ (الثلية الثربية، قسم الفيزياء، جامعة بغداد.
}

الخلاصة

في هذا البحث، جرى تصميم وبناء وتنغيل منظومة تضمين موقع وعرض النبضة لاستخدامها في أجر اء التضمين لليزر

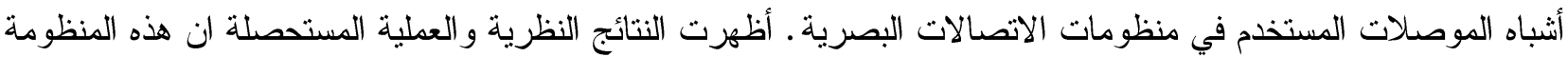
تعمل باستقرارية وموثوقية مقبولين ـ تعتمد هذه المنظومة في عملها على فولتية تشغيل قليلة و إمكانية التحكم بالتردد من خلال التحكم بقيم المقاومة والمتسعة في دائرة امر ار تربط مع هذه المنظومة ـ ـ يعد هذا البحث محاولة لتصميم منظومات التضمين وبنائها على شكل مدمج منل الدائرة المتكاملة لتكون صاحة لترة للاستخدام في معمارية الاتصالات البصرية الحديثة.

لقد وفر اكتشاف الليزر مصدر امثاليا للاشعاع لنضام الاتصالات الضوئية ولم يكن هذا السبب كافيا لاعادة الاهتمام بالطرق الضوئية الني اعيد التفكير بها لسنين وان من الاسباب الاخرى هي بروز الحاجة لانظمة الاتصالات الفضائية التي تتطلب معدات مدمجة ( compact ) قليلة الوزن جعلت حزمة الليزر الضيقة وقليلة الانفراج وذات كثافة القدرة العالية جدا مرشحا مثاليا ـ و على الرغم من

مشاكل الجو والتي لا تظهر في انظمة الفضاء و فان الاتصالات الضوئية الارضية توفر حلا مباش ر رالتحديد عرض النطاق لانظمة التقليدية. يمكن تسمية اكثر انظمة الاتصالات عن بعد (Telecommunication) المعقدة في الوقت الحاضر بانظمة نقطة-الى- نقطة (Point-Point) التي تحمل مختلف القنوات من الهو اتف الى التلفزيون الملون • يمكن ان يصل التزدد الناقل في هذه الانظمة الى HZ 12 X 10 و الاي يناظر موجة مايكروية طولها

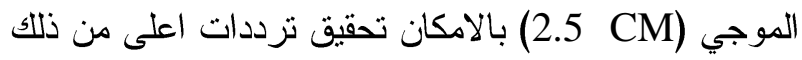
ولكن عند التزدد 24 GH z يكون الامتصاص في الجو كبيرا وعند التردد ( 35 GH z ) تظهر مشاكل عديدة

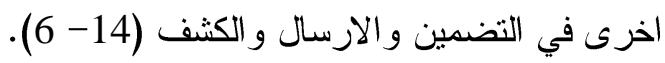

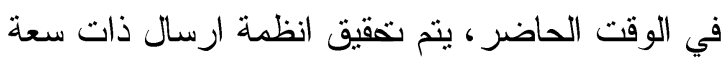

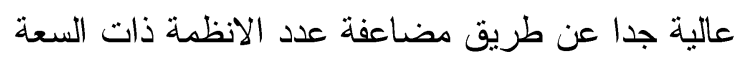

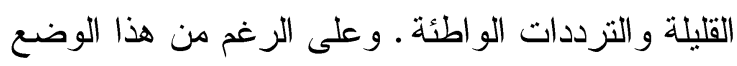

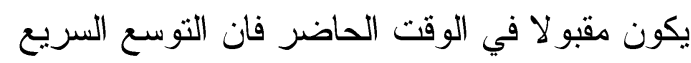

المقدمة

تعتبر دوائر التضمين Modulation من أهم الدوائر الإلكترونية في نظم الاتصالات السلكية ـ وتعتبر الموجات المضمنة أساس عمل البث الإذاعي و التلفزيوني للسهولة التقنية في إرسال الموجات الحاملة ذات الترددات العاليه بالاشعاع (الانتشار) خلال الجو ـ كما ان الموجات المضمنة يمكن اختبار ها من طيف ترددي يحتوي علا موجات أخر ا باستخدام دوائر اختيار إلكترونية وتستخدم الموجات المضمنة أيضا في كثير من المقاييس الالكترونية منل مقياس الانفعال الإلكتروني وفي منضومات القياس عن بعد (Telemetry) الاتصالات الحديثة تستتد بشكل كبير الى الترددات الر اديوية و الموجات المايكروية (RF) الاتصالات الضوئية لعدم كفاية الاجهزة المتوفرة بالاضافة

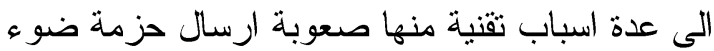
خلال جو الارض، وكون معظم انضمة الاتصالات تعمل

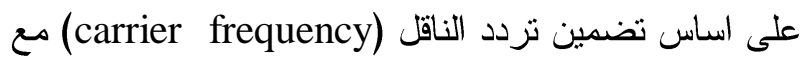
الاشارة بحيث يستخدم واحد للاشعاع لارسال عدد من

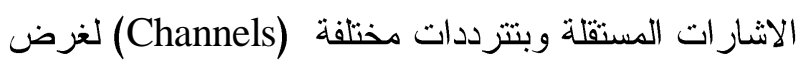
زيارة عدد القنوات المتوفرة يكون من المستحسن تضمين التردد الناقل لاكبر ممكن مقدار 10 \% من التردد الحقيقي. وان صعوبات تضمين موجة الضو ء الى هذه الدرجة تكون

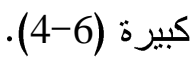


عند اجر اء النمذجة على الاشارة مقارنة بنبضة ضيقة

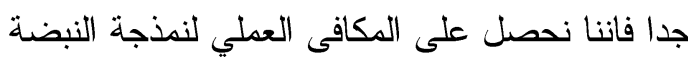
هذه النماذج يمكن استخدامها لتضمين الموقع (IMPUSE)

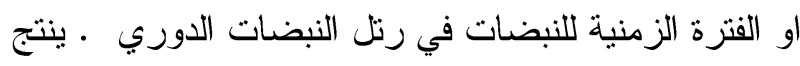
تضمين مدة النبضة (PDM) عند تغير وقت حدوث الحافتين المتقدمة والمتاخرة او احداهما نسبة لاشتارة النطاق الاساس. وتسمى هذه العملية بتضمين موقع النبضة .(17-18) (PPM)

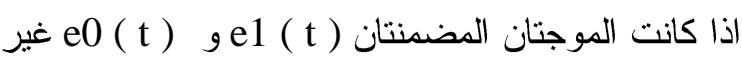

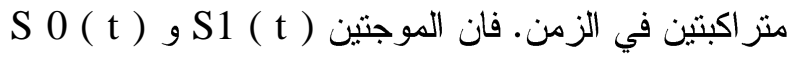

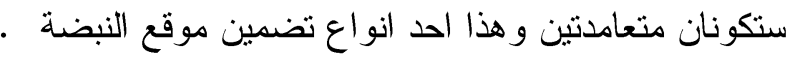
لندرس النبضات المضمنة ذات الاثكلكل الاتية (2-1):

$$
\begin{aligned}
e_{1}(t) & =\left(\frac{4 E}{T}\right)^{\frac{1}{2}} & & 0<t \leq \frac{T}{2} \\
& =0 & & \frac{T}{2}<t \leq T \\
e_{o}(t) & =0 & & 0<t \leq \frac{T}{2} \\
& =\left(\frac{4 E}{T}\right)^{\frac{1}{2}} & & \frac{T}{2}<t \leq T
\end{aligned}
$$

يلعب التضمين دور ا مهما سفي نضم الاتصالات عموما

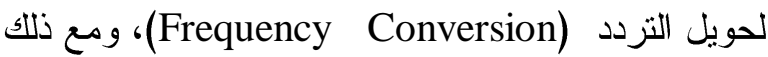
فان له اغر اض اخرى منها تخصيص موقع الترددات Bandwidth ) (FLA)

(Alteration المسمو عة (Audio) لها مركبات تردد بحدود $100 \mathrm{~Hz}$

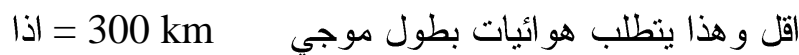

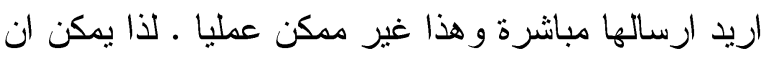
يتم اختزال طول الهو ائي الى 300 m = باز احة هذا التزدد الى MH z 1 1 كما يمكن استخدام الضمين لتقليل الضوضاء و التداخل اذ ان انو اعا معينة من التضمين

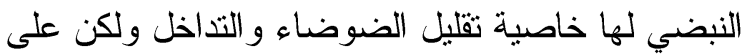
حساب عرض حزمة الارسال (معدل التزدد) وذلك يحتح زيادة عرض الحزمة الاصلية ـ ويمكن التغلب على تحديدات الاجهزة اعتمادا على التضمين اذ يكون تصميم نظام معين

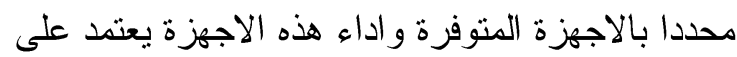

و المستمر في الاتصالات يدعو الى ايجاد حل اخر ـوان

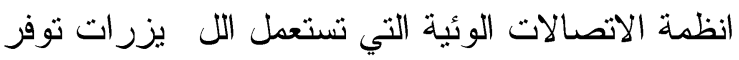

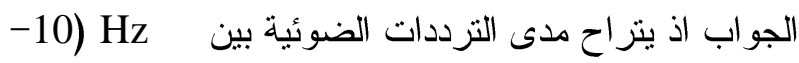
10)، لذلك فان تضمينا بمقدار (\% (0.1) يعني زيادة هائلة

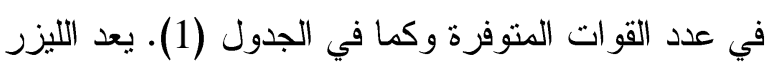
مثاليا للاتصال اذا كان يمنلك قدرة خرج مستمرة وكبيرة ومتانة عالية وعمر طويل و امكانية العمل بدرجة حرارة

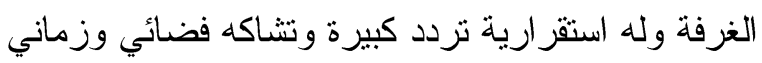

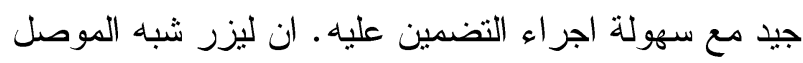

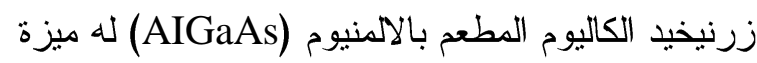
منفردة في ذلك لسهولة التضمين بمقدار بـ 10 عن طريق تضمين تيار الدخل المستمر ، كذللك له عمر تشغيل طويل جدا، سومع ذلك فانه يعاني من صغر التشاكه الفضائي

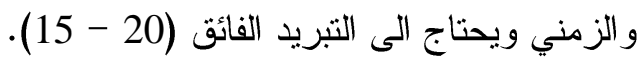

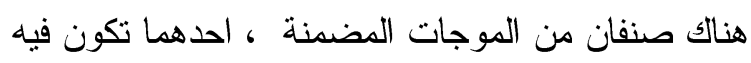

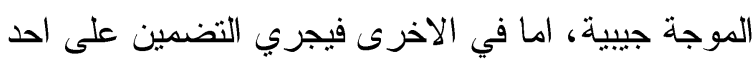

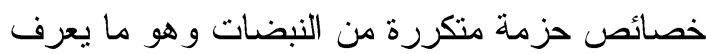
بالتضمين الزنضي (Pulse Modulation) فاذا اعنبرنا اثنارة موجة حاملة كما في المعادلة الاتية :

$\mathrm{E}(\mathrm{t})=\mathrm{E} \sin 0(\mathrm{t})$

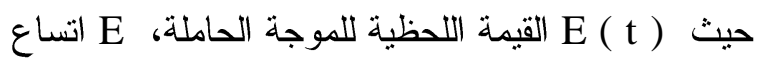
الموجة ، ( t ) م الاز احة الطورية و t الزمن. يمكن تعريف التضمين على انه عملية تغير احد معلمات (Parameters ) عادة تكون جييية ( Sinusoidal ) وفقا لاشارة المعلومات

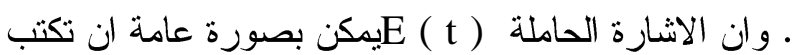

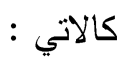

$$
E(t)=A c \cos (2 \pi f c-\theta c)
$$

حيث ان Ac يمتل الاتساع، Fc تمثل التزدد، Oc تمثل الطور . ان التضمين النبضي يمكن ان ياخذ اثكال مختلفة

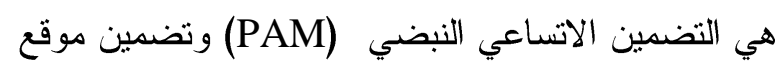

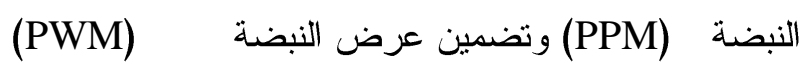
و التضنين النبضي الجفري(PCM). 


$$
\text { لاستخدام هذه الدائرة مع ليزر اثباه الموصلات. }
$$

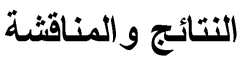

يوضح الشكل (3) نتائج تشغيل الدائرة باستخدام

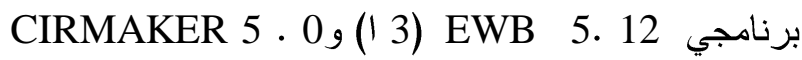

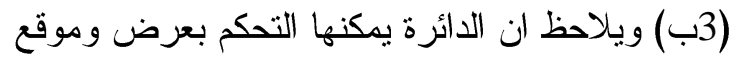
النبضة بشكل جيد مما شجعنا على تتفيذ الدائرة عمليا. يبين الثكل (4) الاثشارة المستخدمة كاثشارة ادخال عن طريق الطرف(5) من الدائرة الاولى (input signal)

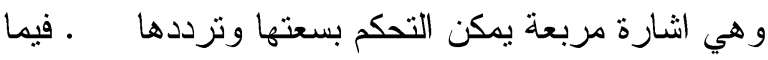
يوضح الثكل (5) التغبر الحاصل في عرض الأنشارة المربعة الخارجة من الدائرة الاولى اذ يزداد عرض الألئ الأشارة بسبب التاخير الحاصل في خروج الاشارة من الدائرة بسبب التاري

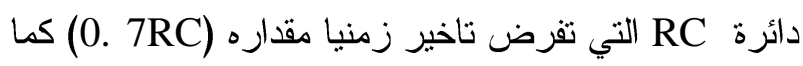

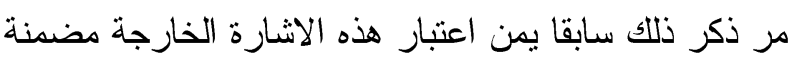

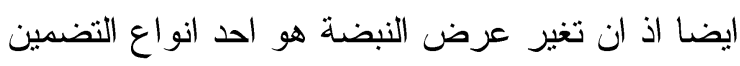

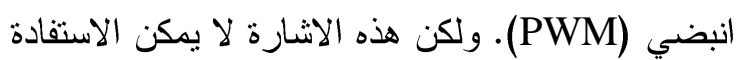
منها بشكل كبير في تضمين ليزر انشباه الموصلات

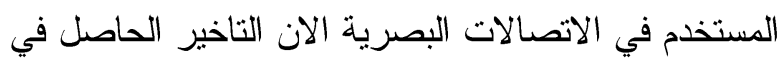
عرض النبضة يمكن ان يسبب تسخين الليزر وكما هو الآئ معلوم فان تر اكيب اثباه الموصلات تككون شديدة الثاثير و الحساسية لاي تغير في درجة حرارة التركيب و هذا يؤثر سلبا على اداء الليزر. يوضح الثكل (6) الاشارة المربعة الداخلة الى الدائرة

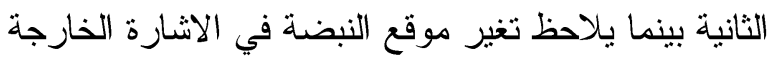

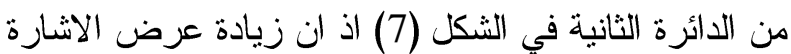

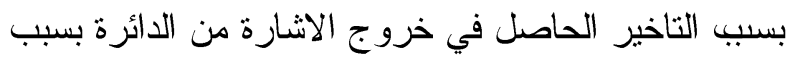

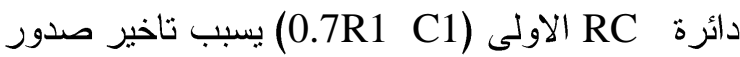
الاشارة الاصلية من الدائرة الثانية وبالتالي تغير موقعها

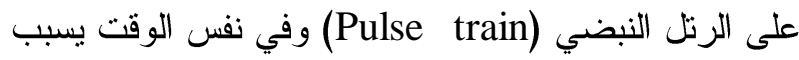
هذا التاخيي صدور الاشتارة النهائية بنفس العرض العضان

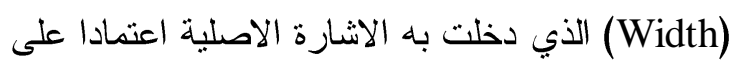

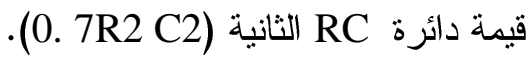
جرى تغير تردد الاثارة الداخلة الى منظومة ليزر الثباه الموصلات عن طريق تضمين قدرة التشغيل وقياس التغير المتوقع في تردد الاشارة الخلرجة والتي هي اثارة الليزر
الترددات المستخذمة وان الضمين يمكن ان ينقل الاثشارة الى ذلك الجزء من طيف التزدد الذي عنده تكون تحديدات الاجهزة اقل ما يمكن(12 و 8 و 5 - 1).
و (EWB 5. 12)

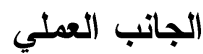
(CIRMAKER 5.0)

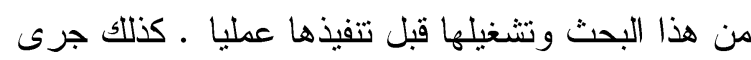
استخدام برنامج (EDS 1. 2) لتشغيل الدائرة و الحصول .(2) عاى جدول الحقيقة لها وكما مبين في الجدول استخدمت نفس البرامج السابقة لمحاكاة اداء الدائرة حيث

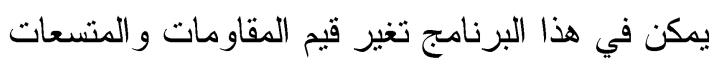
وتردد الاشارة الداخلة والحصول على نتائج النتغيل و الاداء قبل تتفيذ الدائرة عمليا.

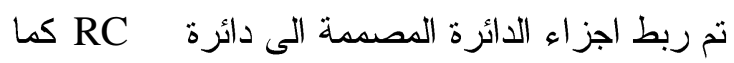
مبين في الثكل (11) لغرض التعرف على شكل الاشارة

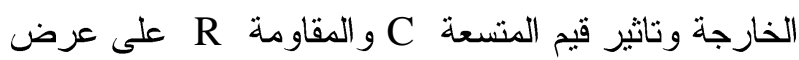

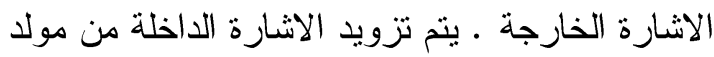
الاشارات (Function generator) حيث يمكن التحكم بشكل الثارة وترددها وسعتها و ادخالها الى الدائرة عن

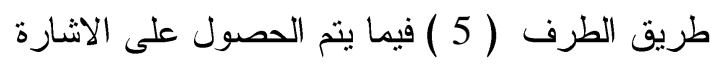

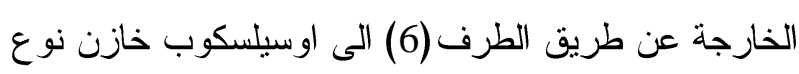
TEKTRONIX 100MHz DIGISCOPE يمكن قياس تردد وسعة وعرض الاثشارة الخارجة والمقارنة ما بين الاشارة الاصلية و الاشارة المضمنة.

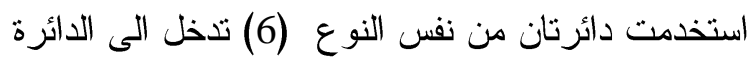

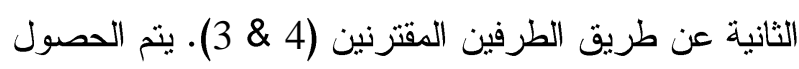

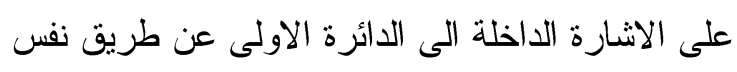
مولد الاشارات المستخدم في الحالة السابقة وكنلك تسجيل الاشثارة الخارجة من الدائرة الثانية على نفس جهاز الاوسيلسكوب. ججرى بعد ذلك تجميع اجز اء الدائرة داخل

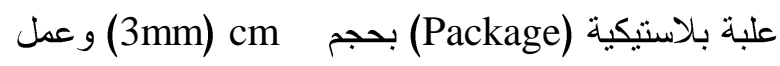
توصيلات خارجية لاطر افها لتمتل دائرة متكاملة بمكن بحن استخدامها بشكل مباشر وموثوق. يمكن استخدام الاشارة الخارجة من دائرة التضمين هذه في تثغيل ليزر اثباه الموصلات المستخدم في الاتصالات البصرية والذي يحتاج فولتية تنثغيل تتزاوح ما بين التين 
جدول (1) مقارنة عدد قنوات الهاتف والتلفزيون التي

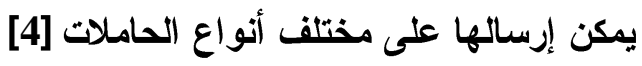

\begin{tabular}{|c|c|c|c|c|}
\hline $\begin{array}{c}\text { Carrier } \\
\text { Type }\end{array}$ & Frequency Range & $\begin{array}{c}\text { Usable } \\
\text { Bandwidth }\end{array}$ & $\begin{array}{c}\text { Approximate } \\
\text { Number of } \\
\text { Telephone } \\
\text { Channels }\end{array}$ & $\begin{array}{c}\text { Approximate } \\
\text { Number of TV } \\
\text { Channels }\end{array}$ \\
\hline \hline $\mathrm{LW}$ & $30 \mathrm{kHz}-300 \mathrm{kHz}$ & $10 \%$ & 3 & - \\
\hline \hline $\mathrm{MW}$ & $300 \mathrm{kHz}-3 \mathrm{MHz}$ & $10 \%$ & 25 & - \\
\hline \hline SW & $3 \mathrm{MHz}-30 \mathrm{MHz}$ & $10 \%$ & 200 & - \\
\hline \hline $\mathrm{VHF}$ & $30 \mathrm{MHz}-300 \mathrm{MHz}$ & $10 \%$ & 4000 & - \\
\hline \hline UHF & $300 \mathrm{MHz}-3000 \mathrm{MHz}$ & $10 \%$ & 10.000 & 10 \\
\hline \hline Microwave & $3000 \mathrm{MHz}-10^{12} \mathrm{~Hz}$ & $10 \%$ & 100.000 & 100 \\
\hline \hline Optical & $5.10^{13} \mathrm{~Hz}-10^{1 \mathrm{H}} \mathrm{Hz}$ & $0.1 \%$ & $10^{8}$ & $10^{3}$ \\
\hline \hline
\end{tabular}

جدول (2) جدول الحقيقة للائرة المتكاملة المصصمة في هذا البحث باستخدام برنامج EDS1.2

\begin{tabular}{|c|c|c|c|}
\hline \multicolumn{3}{|c|}{ Input conditions } & \multirow{2}{*}{$\begin{array}{l}\text { Output } \\
\text { mode }\end{array}$} \\
\hline Al & A2 & $\mathbf{B}$ & \\
\hline 1 & 1 & Any & Inhibit \\
\hline Any & Any & 0 & Inhibit \\
\hline$\downarrow$ & 1 & 1 & Trigger \\
\hline 1 & $\downarrow$ & 1 & Trigger \\
\hline$\downarrow$ & $\downarrow$ & 1 & Trigger \\
\hline 0 & 1 & $\uparrow$ & Trigger \\
\hline 1 & 0 & $\uparrow$ & Trigger \\
\hline 0 & 0 & $\uparrow$ & Trigger \\
\hline
\end{tabular}

على الكاشف الضوئي ، وقد اوضحت النتائج المبينة في الشكل(8) ان تردد الاشارة الخارجة لا يعتمد على تردد

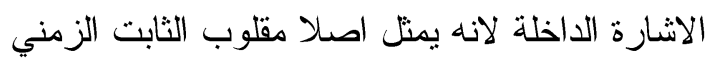
الذي يتحدد بدوره بقيمتي المقاومة و المتسعة في (t= RC)

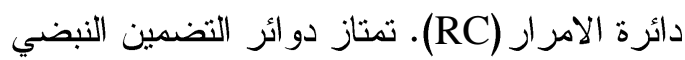

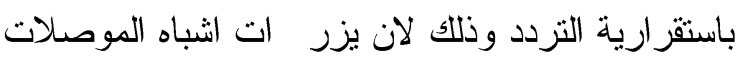

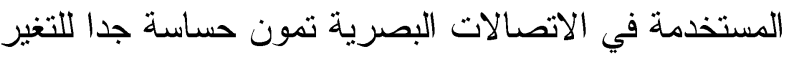
في تردد اشارة التضمين ـ اذا كانت خصائص الدائرة

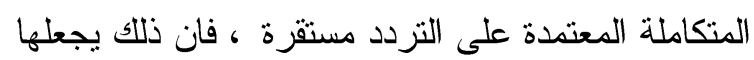
مؤهلة اكثر للاستخدام بشكل واسع في دوائر الاتصالات و التضمين. جرى تغير قيمة المتسعة(C2) قياس تاثير ذلك على قيمة الثابت الزمني RC لكل من الدائرتين وبالتالي تاثير ذلك دئي على تردد الاشارة الخاجة من الدائرة ـ ـ الثكل(9) بين ثاثير باثير تغير قيمة المتسعة على تردد الاشارة الخرجة من الدائرة

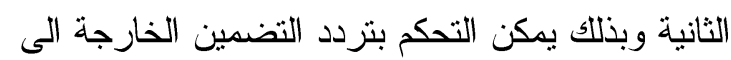

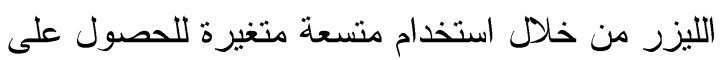
القيمة المطلوبة لتردد التضمين. وجد عمليا ان القيم المتلى لمتسعة دائرة الامرار تتراوح

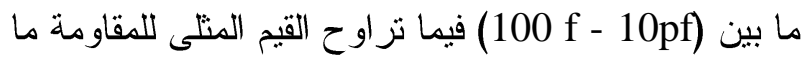

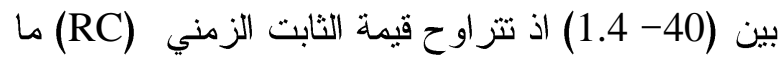
بين (2. 8S - 9.8NS). الاستنتبجات

من النتائج المستحصلة، نستتنج انه يمكن تصميم دائرة

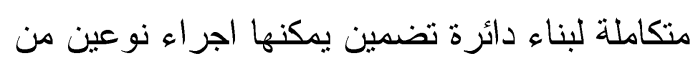
التضمين النبضي في وقت واحد هما تضمين عرض لفره النبضي (PWM) وتضمين موقع النبضة (PPM) ـ ميكن

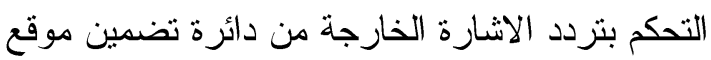
النبضة من خلال التحكم بقيم المتسعة و المقاومة في دائرة

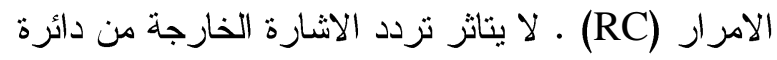

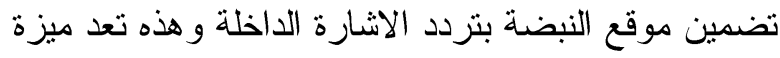
لمثل هذه الدوائر يمكن استخدام هذه الدائرة لتضمين ليزرات اثباه الموصدات المستخدمة في الاتصالات اليصرية اذ يمكن لهذه الدو ائر ان تعمل بفولتيات قليلة كالاتي تعمل بها هذه الليزرات. 

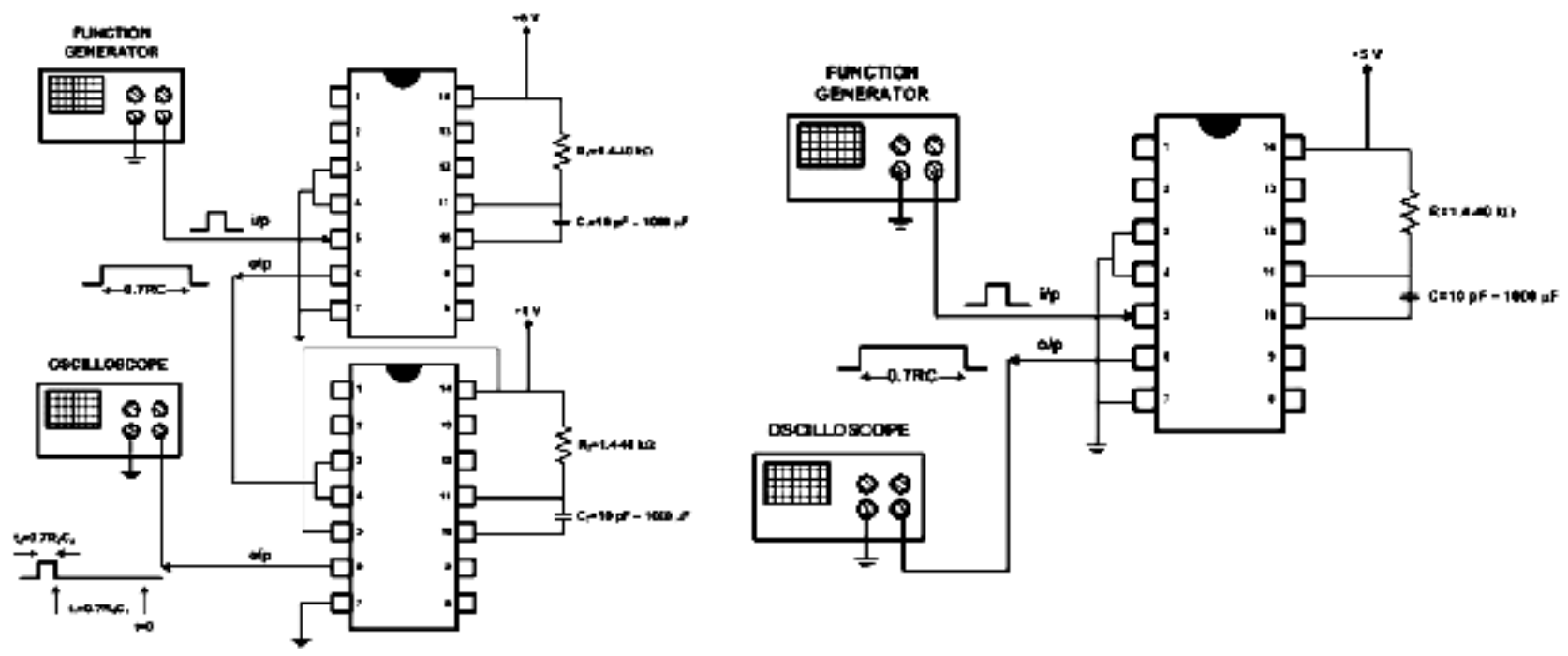

\section{(ب)}

شكل (1) الترتيب العملي لقياس تردد وسعة وعرض الإشارة الخارجة

(أ) باستخدام دائرة واحدة و (ب) باستخد ام دائرتين

\section{SEMICONDUCTOR \\ LASER POWER \\ SUPPLY}

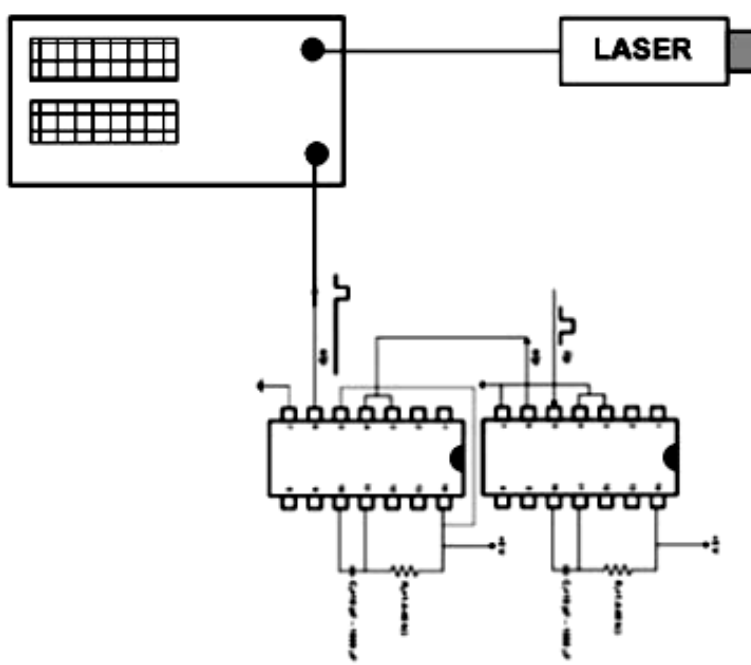

شكل (2) الترتيب العملي لتضمين موقع النبضة لليزر أثباه الموصلات 


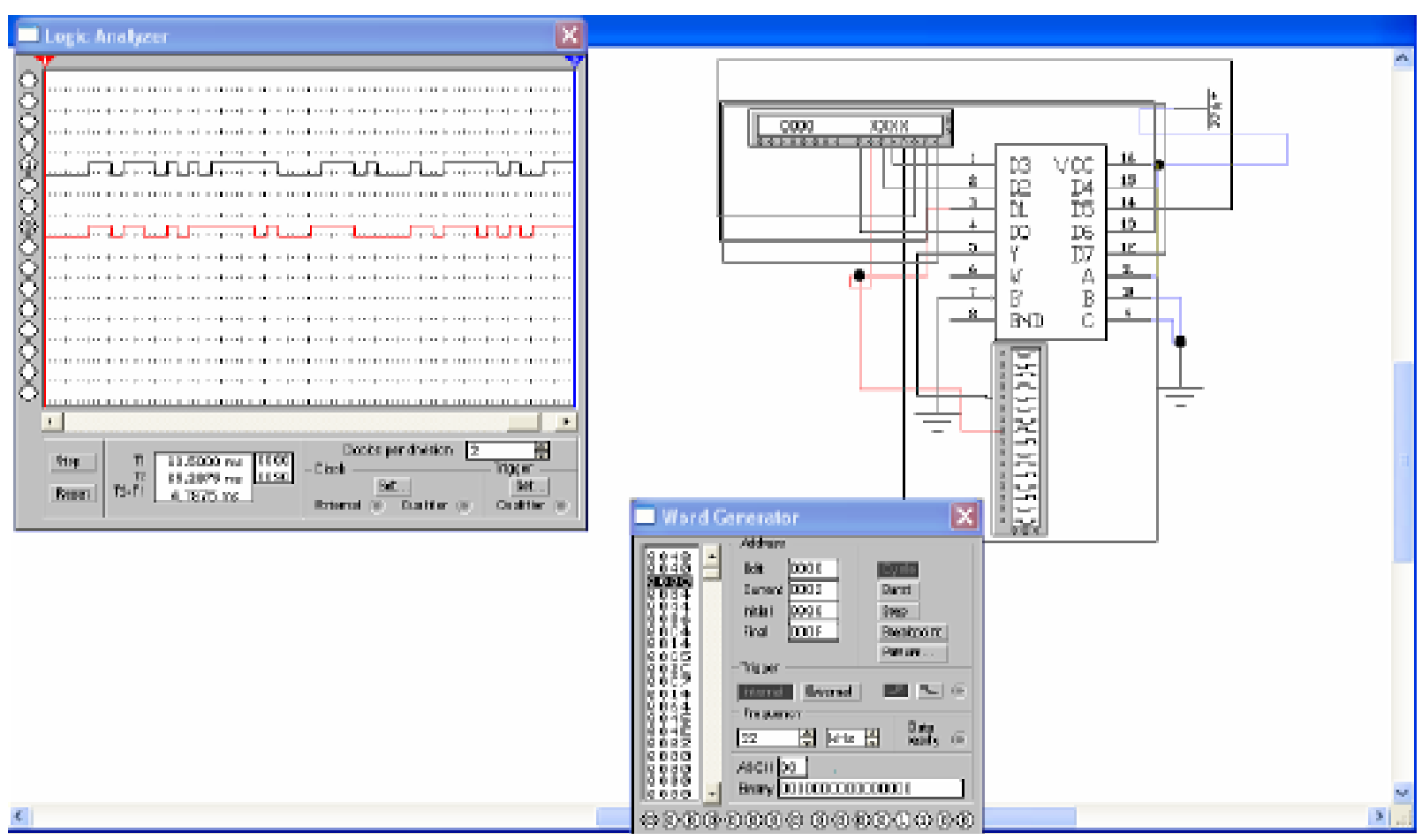

(i)

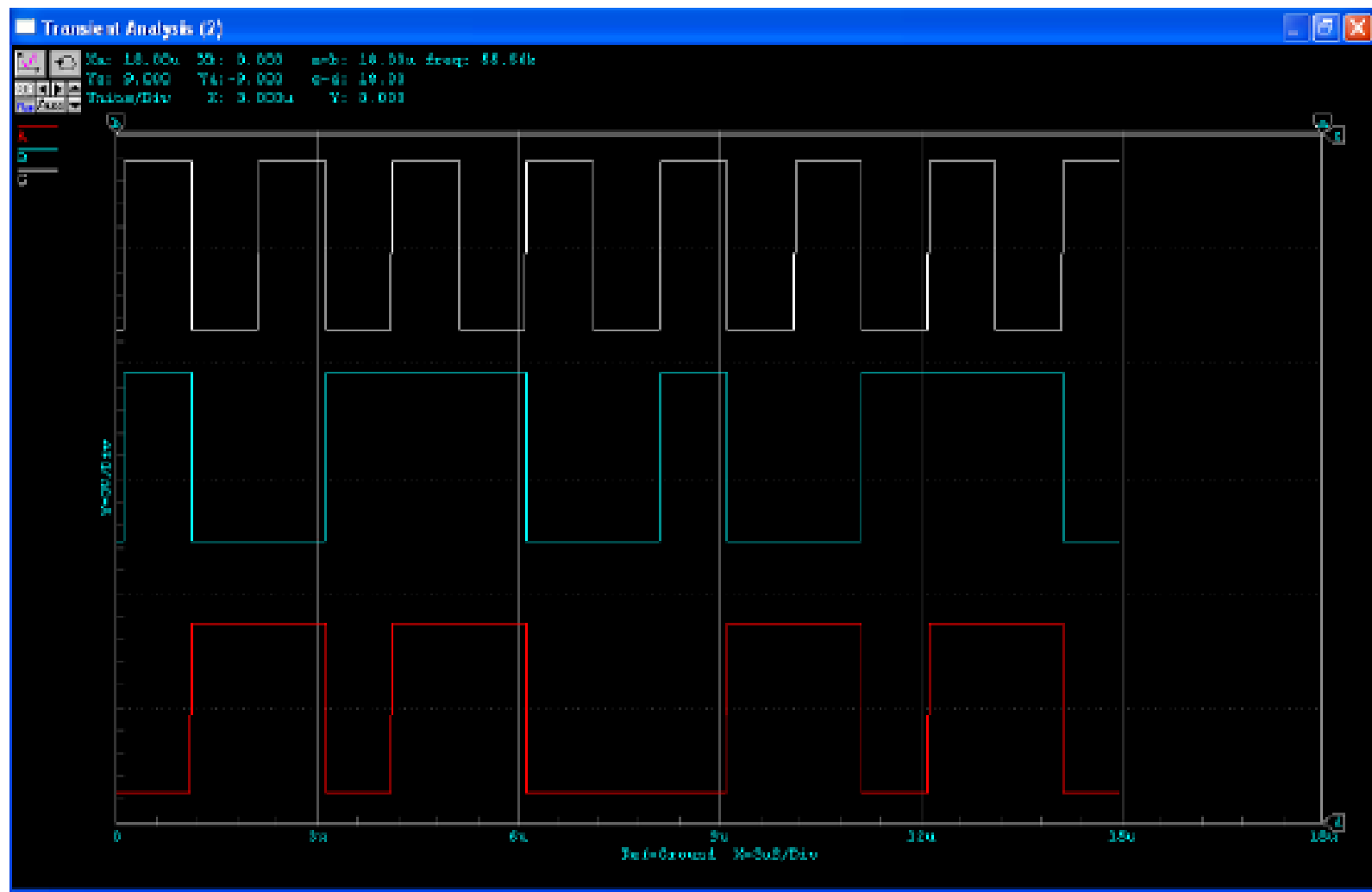

(ب)

شكل (3) نتائج تثغيل الائرة المتكاملة المصممة في هذا البحث باستخدام

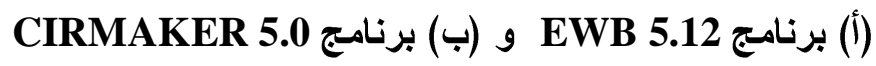




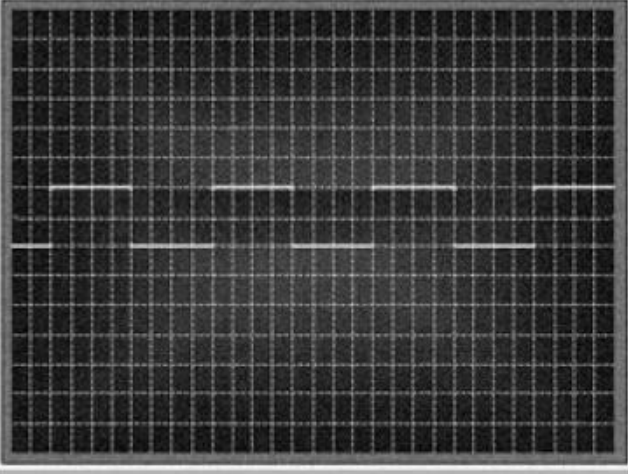

شكل(5) شكل الإشارة الخارجة في حالة استخدام دائرة واحدة

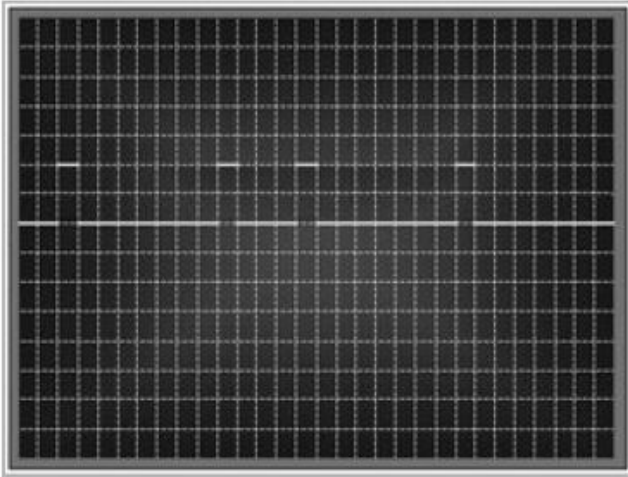

شكل (7) شكل الإثارة الخارجة من الائرة الثانية

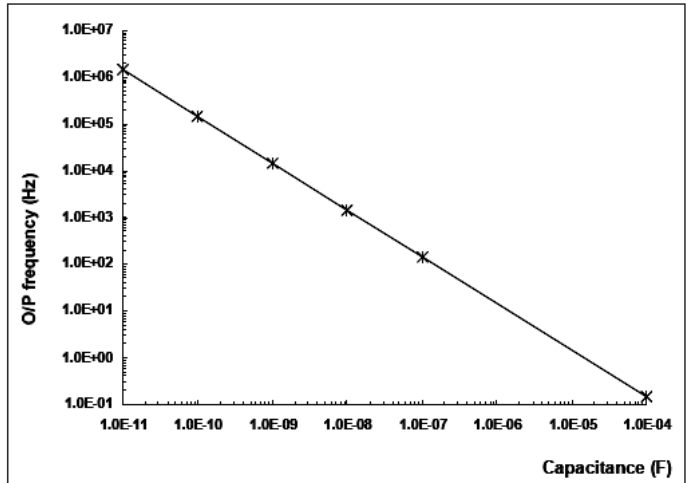

شكل (9) تغير تردد الإثارة الخارجة مع تغير سعة المتسعة في دائرة RC الثاتية

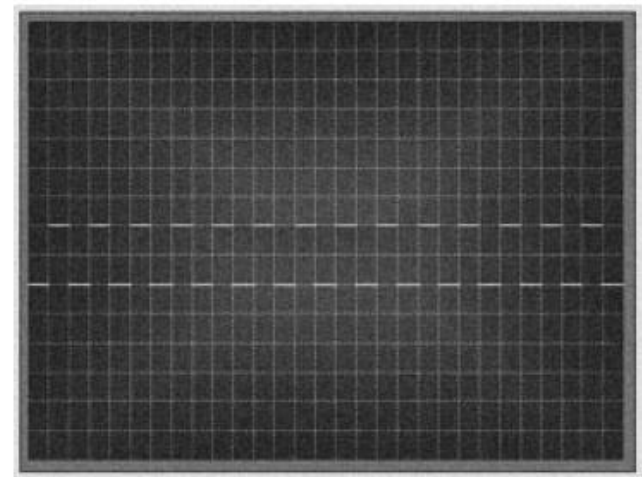

شكل(4) الاشارة الداخلة الى الائرة المتكاملة من مولد الإشـارات

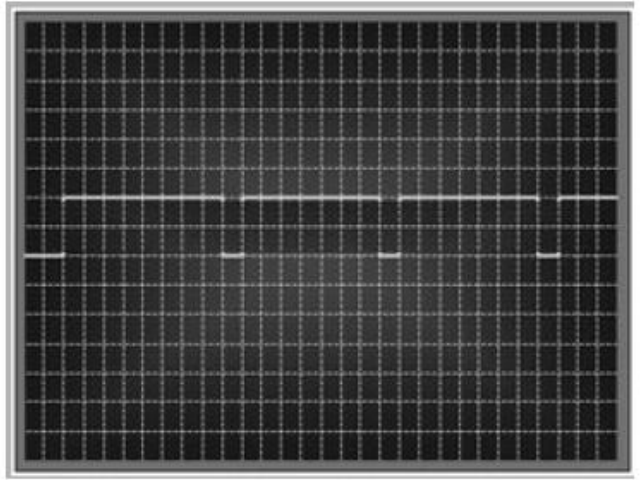

شكل (6) شكل الإشارة الاخلة الى الدائرة الثانية

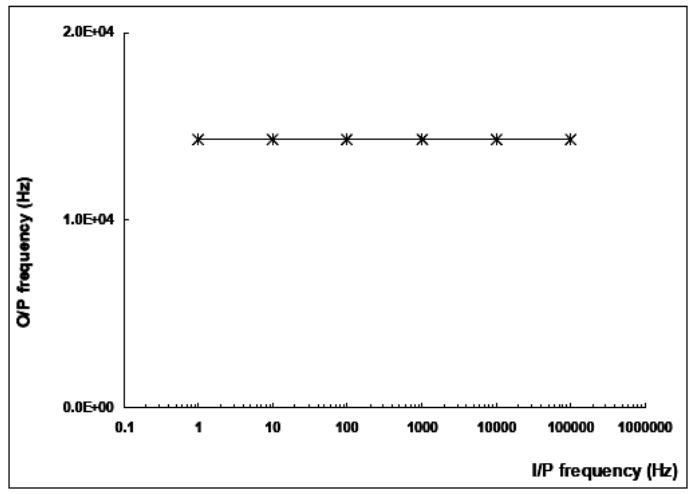

شكل (8) ثبوت تردد الإشارة الخارجة مع تغير تردد الداخلة
[5] A. Yariv, "Optical Electronics in Modern Communications", 5th edition, Oxford University Press (NY), 1997.

[6] A.G. Williams and E.F. Lewise, "Characterization of cyclostationary random signal processes," IEEE Transactions on Information Theory, Vol. IT-21, 1975, p. 1.

[7] Y. Ueno, Y. Ohgushi and T. Yasugi, "An optical fibre cable communication system using pulse-interval-modulation," IEE 1st European Conf. on Optical Fibre Communication, 1975, pp 156-158.

\section{المصادر - اد}

[1] M.H. Jones, "A Practical Introduction to Electronic Circuits", 2nd edition, Cambridge University Press (UK), 1985.

[2] J. M. Senior, "Optical Fiber Communications: Principles and Practice", Prentice Hall International (UK), 1985.

[3] F.G. Stremler, "Communication Systems", 3rd Edition, Addison Wesley, 1992, pp 168- 176, pp 130-145.

[4] M.M. Liu, "Principles and Applications f Optical Communications", McGraw-Hill Co., Inc., 1996. 
[18] D. Shiu, and J. Kahn, Differential PulsePosition Modulation for Power-Efficient Optical Communication, IEEE Transactions on Communications, Vol. 47 No. 8, 1999, p. 1201.

[19] J. Loughry and D.A. Umphress, Information Leakage from Optical Emanations, ACM Transactions on Information and System Security, Vol. 5, No. 3, 2002, pp. 262-289.

[20] U. Sethakaset and T. Aaron Gulliver, Differential Amplitude Pulse-Position Modulation for Indoor Wireless Optical Communications, European Journal on Applied Signal Processing, Vol. 1, 2005, pp. 3-11.

\begin{abstract}
In this work. A PPM-PWM system designed. constructed and operated to be employed for modulation of semiconductor lasers used in optical communication system The simulation and experimental resultsobtained from this work-showed that this modulation system has acceptable stability and reliability. this system is operated at low voltages and the output frequency can be controlled by according to the values of resistors and capacitors in the passing circuit connected to this system. This work is an attempt to design modulation systems and make them compact as the integrated circuit to be suitable for the modern optical communications architectures.
\end{abstract}

[8] M. Sato, M. Murata and T. Namekawa, "Pulse interval and width modulation for video transmission," IEEE Trans. on Cable Television, Vol. CATV-3, 1978, pp. 165173.

[9] A. Okazaki, "Pulse interval modulation applicable to narrowband transmission," IEEE Transactions on Cable Television; Vol. CATV-3, 1978, pp. 155-164.

[10] Y. Ueno and T. Yasugi, "Optical fibre communication systems using pulseinterval modulation," NEC Research \& Development, 1978, pp. 45-52.

[11] A. Okazaki, "Still picture transmission by pulse-interval modulation, IEEE transactions on cable television", Vol. CATV-4, 1979, pp. 17-22.

[12] N.M. Calvert, M.J.N. Sibley and R.T. Unwin, "Experimental optical fibre digital pulseposition modulation system," Electronic Letters, Vol. 24, No. 2, 1988, pp 129-131.

[13] B. Wilson and Z. Ghassemlooy, "Pulse time modulation techniques for optical fibre communications: a review," IEE Proc. J, Vol. 140, 1993, pp. 346-357.

[14] J. Elmirghani and R. Cryan, "Analytic and numeric modelling of optical fibre PPM slot and frame spectral properties with application to timing extraction," IEE Proc. on Commun., Vol. 141, No. 6, 1994, pp. 379-389.

[15] Z. Ghassemlooy, E. Kaluarachchi, R. Reyher and A. Simmonds, "A new modulation technique based on digital pulse interval modulation for optical-fibre communication," Microwave and Optical Technology Letters, Vol. 10, N. 1, 1995, pp. 1-4.

[16] D.C. M. Lee, J.M. Kahn, and M.D. Audeh, Trellis-Coded Pulse-Position Modulation for Indoor Wireless Infrared Communications, IEEE Transactions on Communications, Vol. 45 No. 9, 1997, p. 1080.

[17] V. Vilnrotter, M. Simon and T. Yan, The Power Spectrum of Pulse-Position Modulation With Dead Time and Pulse Jitter, TMO Progress Report 42-133 May 15, 1998. 